\title{
EFECTO DE TRES DENSIDADES DE CULTIVO EN CONDICIONES DE LABORATORIO DE ALEVINOS DE PAICHE ARAPAIMA GIGAS SOBRE SUS PARÁMETROS HEMATOLÓGICOS, BIOQUÍMICOS SANGUÍNEOS Y BIOMÉTRICOS
}

\author{
José DELGADO RAMÍREZ ${ }^{1}$, Carmela REBAZA ALFARO² ${ }^{2}$ Daniel PAREDES LÓPEZ ${ }^{3 \mathrm{a}}$, Rizal ROBLES \\ HUAYNATE $^{3 b}$, Roger BAZÁN ALBITES ${ }^{2}$ \\ 1 Tesista de la Universidad Nacional Agraria de la Selva - UNAS, Facultad de Zootecnia. Huánuco, Perú. E-mail: \\ jose_ramdel@hotmail.com. \\ 2 Instituto de Investigaciones de la Amazonía Peruana (IIAP). Programa de investigación para el uso del agua y \\ conservación de sus recursos (AQUAREC). Carretera Federico Basadre km 12.4 Pucallpa, Perú. Telefax: 061-573732. E- \\ mail:iiapuc@iiap.org.pe.Pucallpa, Perú. \\ 3 Universidad Nacional Agraria de la Selva - UNAS. Facultad de Zootecnia. Huánuco, Perú. Email: \\ daniel.paredes9@gmail.com (a); Email: rizalcides@gmail.com (b).
}

\begin{abstract}
RESUMEN
Se evaluó el efecto de tres densidades de cultivo de alevinos de Arapaima gigas en condiciones de laboratorio, sobre los valores hematológicos, perfil bioquímico sanguíneo y parámetros biométricos. Ciento sesenta y ocho alevinos de paiche con peso y talla inicial de $75.06 \pm 10 \mathrm{~g}$ y $22.69 \pm 1.2 \mathrm{~cm}$, respectivamente, fueron distribuidos al azar en 3 tratamientos $\left(\mathrm{T} 1=7, \mathrm{~T} 2=14, \mathrm{y} \mathrm{T} 3=21\right.$ alevinos $\left./ 56 \mathrm{~L}^{-1}\right)$ por cuadruplicado. Los peces fueron alimentados con una dieta extruida (50\% PB), empleando una tasa de alimentación equivalente al 3\% de su biomasa corporal. Los parámetros biométricos se registraron cada 10 días, mientras que la toma de muestras para evaluar los parámetros sanguíneos en los días 0,33 y 63 del experimento. Los valores de hematocrito, hemoglobina, glucosa, triglicéridos, aspartato aminotransferasa, así como el consumo diario de alimento, ganancia de peso e incremento de talla, no mostraron diferencias entre tratamientos $(\mathrm{P}>0.05)$. Las concentraciones de proteína, albúmina, colesterol, alanina aminotransferasa y los parámetros biométricos de conversión alimenticia aparente y factor de condición, mostraron diferencia entre tratamientos $(\mathrm{P}<0.05)$. Los valores de hematocrito y hemoglobina, así como las concentraciones de glucosa, proteína, colesterol, triglicéridos y alanina aminotransferasa, presentaron diferencias significativas $(\mathrm{P}<0.05)$ con respecto a la edad. Se concluye que las densidades de cultivo evaluadas no causaron efecto en los parámetros biométricos y sobre los valores hematológicos, con excepción de la conversión alimenticia y el factor de condición, en tanto que las concentraciones del perfil bioquímico sanguíneo se ven afectadas parcialmente.
\end{abstract}

PALABRAS CLAVE: alevino, densidad, paiche, parámetros hematológicos.

\section{EFFECT OF THREE DENSITIES OF CULTURE UNDER LABORATORY CONDITIONS OF PAICHE Arapaima gigas FINGERLINGS ON ITS HEMATOLOGICAL AND BIOCHEMICAL PROFILES AND BIOMETRIC PARAMETERS.}

\section{ABSTRACT}

The aim of this study was to evaluate the effect of three stocking rates of Arapaima gigas fingerlings under laboratory conditions on hematological values, blood biochemical profile and biometric parameters. One hundred sixty eight fingerlings of $75.06 \pm 10 \mathrm{~g}$ and $22.69 \pm 1.2 \mathrm{~cm}$, respectively, were distributed at random into three treatments $\left(\mathrm{T} 1=7, \mathrm{~T} 2=14\right.$ and $\mathrm{T}=21$ fingerlings $\left./ 56 \mathrm{~L}^{-1}\right)$ with 4 replicates were used. Fish were fed with an extruded diet $(50 \% \mathrm{CP})$ using a feeding rate equivalent to $3 \%$ of body biomass. The biometric parameters were recorded every ten days, meanwhile blood samples were collected at days 0,33 and 63 of the experiment. The hematocrit, hemoglobin, glucose, triglycerides, aspartate aminotransferase, daily feed intake, weight gain and height increase did not show differences among treatments $(P>0.05)$. The concentrations of total protein, albumin, cholesterol, alanine aminotransferase, feed conversion, and condition factor shown difference among treatments $(\mathrm{P}<0.05)$. The hematocrit and hemoglobin, as well as the concentration of glucose, total protein, cholesterol, triglycerides and alanine aminotransferase did show statistical difference $(\mathrm{P}<0.05)$ with respect to fish age. It is concluded that the stocking rates densities caused no effect on the biometric parameters and hematological values except for feed conversion and the condition factor while the blood biochemical profile were partially affected.

KEYWORDS: fingerling, density, paiche, hematological parameters. 


\section{INTRODUCCIÓN}

El estudio está relacionado con la ictiohematología, disciplina que estudia los componentes sanguíneos en peces, morfología, bioquímica y fisiología. Asimismo, genera información sobre diferentes aspectos del metabolismo, órganos y sistemas del individuo, desbalance nutricional, efectos tóxicos, condiciones anóxicas, presencia de enfermedades infecciosas y otros agentes estresantes, constituyéndose en una herramienta útil en la determinación del estado de salud de peces en cultivos intensivos (Centeno et al., 2007; Hahn-Von et al., 2011; Tórrez et al., 2012).

El paiche Arapaima gigas es una especie nativa de la cuenca amazónica, es considerado el pez de escamas más grande de agua dulce, existiendo ejemplares que sobrepasan los $200 \mathrm{~kg}$ de peso y alcanzan hasta $3 \mathrm{~m}$ de longitud. Considerado el gigante del Amazonas, goza de renombre internacional y tiene un gran potencial para conquistar los mercados del mundo (Franco et al., 2007); Alcántara et al., 2006).

El cultivo de paiche en ambientes controlados involucra necesariamente a la producción de alevinos, ya que con frecuencia son manejados en laboratorio, estanques de tierra y jaulas flotantes, observándose los mejores resultados en condiciones de laboratorio (Padilla et al., 2003); Brandão et al., 2004).

La densidad de crianza es un factor muy importante a tener en cuenta en la acuicultura. Durante el proceso de cultivo a altas densidades, se generan cambios desfavorables tanto en el aspecto nutricional, como en la calidad del agua y la presencia de agentes patógenos. Asimismo, la alta densidad de cultivo, activa factores estresantes en los alevinos, alterando su físiología y condicionando los parámetros biométricos, los valores hematológicos y el perfil bioquímico sanguíneo. Estos indicadores son relevantes, ya que permiten evaluar el grado de influencia de los factores internos y externos en el bienestar de los alevinos (Sagratski et al., 2003; Tavares et al., 2009).

La alta densidad hace variar negativamente los parámetros de calidad del agua, causando modificaciones fisiológicas en los parámetros sanguíneos de los peces, disminuyendo la capacidad de los glóbulos rojos para captar oxígeno, causando hemorragia hepática, irritando las branquias, lo que degenera en una hiperplasia branquial, provocando alteraciones en la osmorregulación, favoreciendo la pérdida de sales y por último, haciendo variar el metabolismo y afectando los parámetros biométricos, lo que conlleva a una reducción de la producción (Hipher, 1993; Ocampo y Ocampo, 1999; Granado, 2000; Asmine y Díaz, 2004; Blanco, 2005; Ruales et al., 2009).
El estrés provocado por la alta densidad de cultivo es un factor que se minimiza fácilmente si se utilizan buenas técnicas de alimentación y se proporciona a cada individuo la ración que le corresponde, disminuyendo, de esta forma, la agresividad entre individuos. Los valores hematológicos y el perfil bioquímico sanguíneo pueden ser afectados por las características nutricionales de la dieta (Toro et al., 2012; Tórrez et al., 2012).

Los eritrocitos son las células más abundantes en la circulación sanguínea y abastecen de oxígeno a las células del organismo, su buen funcionamiento puede ser desequilibrado por altas concentraciones permanentes de catecolaminas, produciendo alteración en los iones del medio celular (Barandica \& Tort, 2008; Tavarez et al., 2009). El hematocrito y la hemoglobina aumentan durante el primer año de vida, debido a que el metabolismo del pez es mayor en su crecimiento y en el periodo de desarrollo de los tejidos hematopoyéticos. Asimismo, los peces amazónicos presentan mayor porcentaje de hematocrito en comparación a los peces de agua salada (Sanz et al., 2001; Fonseca et al., 2010).

Los componentes del perfil químico sanguíneo son indicadores eficientes de los desórdenes fisiológicos, mantienen la presión coloidosmótica en la sangre, siendo influenciados por la edad, el sexo, la calidad nutricional del alimento y la calidad del agua (Hipher, 1993; Castellanos et al., 2003; Asmine \& Díaz, 2004; Herrera, 2004; Tavares et al., 2007; Marcano, 2011).

El presente estudio tiene como objetivo generar información relativa al efecto de las altas densidades de cultivo en alevinos de paiche Arapaima gigas sobre los valores hematológicos, perfil químico sanguíneo, parámetros biométricos y calidad del agua, indicadores que pueden evidenciar el alto potencial de esta especie en cultivos intensivos.

\section{MATERIALY MÉTODOS}

El trabajo de investigación se llevó a cabo en las instalaciones del laboratorio de manejo de alevinos de paiche de la Estación Experimental del Instituto de Investigaciones de la Amazonía Peruana (IIAP)Ucayali, en el kilómetro 12.4 de la carretera Federico Basadre, en el Distrito de Yarinacocha, Provincia de Coronel Portillo, Región de Ucayali, entre las coordenadas $80^{\circ} 24^{\prime} 03^{\prime \prime} \mathrm{S}$ y $74^{\circ} 38^{\prime} 20^{\prime \prime} \mathrm{O}$, a una altitud de $158 \mathrm{msnm}$. La zona pertenece al ecosistema de bosque húmedo tropical, con precipitación media anual de $1,369 \mathrm{~mm}$, temperatura media anual de $25,8^{\circ} \mathrm{C}$ y humedad relativa de $87 \%$.

\section{TRATAMIENTOS}

Se utilizaron 168 alevinos de paiche, obtenidos por 
reproducción en ambientes controlados en el IIAPUcayali, con un peso y una longitud inicial promedio de $75.06 \pm 10 \mathrm{~g}$ y $22.69 \pm 1.2 \mathrm{~cm}$ respectivamente. Estos alevinos fueron distribuidos al azar en tres tratamientos $(\mathrm{T} 1=7, \mathrm{~T} 2=14$, y $\mathrm{T} 3=21$ alevinos $\left./ 56 \mathrm{~L}^{-1}\right)$ con 4 repeticiones cada uno de volumen total por recipiente para los tratamientos 1 , 2 y 3 respectivamente.

El estudio se llevó a cabo utilizando como unidades experimentales 12 tinas plásticas de 85 litros de capacidad, con un sistema de recambio de agua a un caudal de $1.7 \mathrm{~L} \mathrm{~min}^{-1}$ durante 45 minutos, cinco veces al día, con intervalo de dos horas.

El alimento suministrado fue una dieta balanceada comercial tipo extruido de $6 \mathrm{~mm}$ de diámetro y $50 \%$ de proteína, con una tasa de alimentación de 3\% de la biomasa total y con una frecuencia de dos horas entre las 8:00 a.m. - 6:00 p.m., durante 63 días.

\section{CALIDADDE AGUA}

El monitoreo de la calidad de agua se realizó después de transcurridas las 2 y las 13 horas del último recambio. Se registraron las concentraciones de amonio, nitrito, oxígeno disuelto, dióxido de carbono, dureza, alcalinidad y $\mathrm{pH}$ a los 12,42 y 62 días experimentales, midiendo y registrando la temperatura todos los días. Se utilizó un equipo de análisis de aguas dulces modelo AQ-2.

\section{MUESTREOS BIOMÉTRICOS}

La ganancia de peso y talla se monitoreó cada 10 días, registrando el peso y talla de la población total. Al final del bioensayo se realizaron los cálculos de consumo de alimento diario, ganancia de peso diario, incremento de talla, conversión alimenticia, factor de condición y producción de biomasa en $\mathrm{kg}$ $\mathrm{m}^{-3}$.

\section{COLECTA DE MUESTRAS DE SANGRE}

Se realizó la colecta de sangre para determinar los valores hematológicos y perfil bioquímico sanguíneo de los alevinos, para lo cual fueron anestesiados a un nivel II (solución de agua con Eugenol U.S.P y alcohol $96^{\circ}$ a razón de 333.3 ppm L ${ }^{1}$ cada uno). Las muestras fueron tomadas por doble punción en la arteria caudal, haciendo uso de 2 jeringas de $3 \mathrm{ml} \mathrm{y} 2$ agujas de tuberculina por alevino. Las muestras para hematocrito y hemoglobina se colectaron con jeringas impregnadas con heparina como anticoagulante. Se realizaron tres colectas de muestra de sangre $(0,33$ y 63 días experimentales), capturando 4 (muestreo 1) y 12 (muestreo 2 y 3 ), en total se extrajo sangre de 28 alevinos y se tomaron 56 muestras.

\section{ANÁLISIS DE LAS MUESTRAS}

Los análisis de los valores hematológicos se realizaron en un Laboratorio Clínico particular de la ciudad de Pucallpa, Ucayali. El porcentaje de hematocrito se observó por el método de microhematocrito en tubos capilares, según Goldenfarb et al. (1977); citado por Tavares et. al. (1999) y el valor de hemoglobina por el método de la cianometahemoglobina de acuerdo con Collier (1944). Las concentraciones de glucosa, proteína, albúmina, colesterol, triglicéridos, aspartato aminotransferasa y alanina aminotransferasa, fueron determinadas usando un equipo de análisis comercial Wiener Lab (2008) y leídas mediante un espectrofotómetro o analizador bioquímico semiautomatizado en el Laboratorio de Sanidad Animal de la Facultad de Zootecnia en la Universidad Nacional Agraria de la Selva, Huánuco.

\section{ANÁLISIS ESTADÍSTICO}

Para medir el desempeño de los parámetros biométricos se utilizó el diseño completamente al azar, utilizando la co-variable peso y, en el análisis de los parámetros sanguíneos, un Diseño Completamente al Azar con arreglo factorial $3 \times 3$ (tres densidades de cultivo y tres periodos de toma de sangre). Todos los análisis de variancia y comparación de medias se realizaron mediante el programa estadístico Statistical Análisis System (SAS, 1998) y la prueba Tukey con un intervalo de confianza de $95 \%(\mathrm{P}<0.05)$.

\section{RESULTADOS Y DISCUSIÓN}

\section{CALIDAD DE AGUA}

Las características fisicoquímicas del agua se presentan en la Tabla 1. Las concentraciones de amonio, dióxido de carbono, alcalinidad y dureza en los tres tratamientos, son altamente superiores a los reportados por Crescêncio et al. (2005); Padilla et al. (2006); Del Risco et al. (2008) y Fonseca et al. (2010). El oxígeno disuelto es muy inferior, descendiendo hasta cero. Por otro lado, las concentraciones de nitrito no se incrementaron con la misma intensidad que las concentraciones arriba mencionadas, estando condicionadas por factores tales como la ausencia de bacterias nitrificantes, el $\mathrm{pH}$ o el tiempo de recambio de agua (Ono \& Kehdi 2013 Fer 2011). Los niveles de $\mathrm{pH}$ y temperatura no muestran variación relevante y son semejantes a los reportados por Franco et al. (2007); Padilla et al. (2005); Fonseca et al. (2010).

\section{PARÁMETROS BIOMÉTRICOS}

Los resultados de los parámetros biométricos se muestran en la Tabla 2. El CDA, GDP e IDL no 
presentaron variación estadística significativa $(\mathrm{P}>0.05)$ durante el periodo total de evaluación. Joblin (1993), citado por Granado (2000), especifica que la alta densidad de cultivo influye reduciendo el consumo de alimento. Sin embargo, el consumo normal de alimento, según los datos obtenidos en este estudio, puede estar ligado a la presentación física del alimento, a la técnica y la frecuencia de la alimentación, factores que minimizan el estrés producto de la alta densidad de cultivo, como lo especifican Padilla et al. (2003) y Toro et al. (2012), investigaciones con las que concuerdan los resultados del presente estudio. La ganancia de peso diario en los tres tratamientos es superior en contraste a los $2.4 \mathrm{~g}_{\text {día }}{ }^{-1}$ conseguidos por Sagratzki et al. (2003) en alevinos de paiche con edad y peso muy similares, cultivados en jaulas flotantes; son superiores (peso y longitud) a los conseguidos por Del Risco et al. (2008), quienes obtuvieron $4.5 \mathrm{~g}$ y $13.73 \mathrm{~cm}$ de ganancia de peso y longitud respectivamente, en un estudio con una densidad de 1 alevino $50 \mathrm{~L}^{-1}$ en condiciones de laboratorio y con parámetros fisicoquímicos de agua que se acercan a los estándares recomendados para esta especie.

En relación a la condición alimenticia aparente (CAA), existe diferencia significativa $(\mathrm{P}<0.05)$ entre las diferentes densidades. No obstante, los resultados de CAA obtenidos en este estudio son superiores a los obtenidos por Aldea (2002) y Padilla et al. (2003), quienes señalan valores de 1.2 y 2.3 de alevinos respectivamente, cultivados en jaulas flotantes y alimentados con $50 \%$ de proteína en la ración. Por otro lado, Del Risco et al. (2008), consigue 1.07 alevinos. Estos estudios fueron realizados con densidades bajas y calidad de agua muy superior a las utilizadas en este estudio.

El factor de condición $(\mathrm{K})$ muestra variación estadística significativa $(\mathrm{P}<0.05)$ por efecto de la densidad. Estos datos se asemejan a los reportados por Del Risco et al. (2008) de 0.79 alevinos y se distancian de Padilla et al. (2006) (jaulas flotantes), que reporta 0.97 alevinos obtenidos en esta misma especie, edad y tiempo de evaluación. El descenso del factor $\mathrm{K}$ que presenta el $\mathrm{T} 1$ no es producto de la densidad ni calidad del agua, debido a que este tratamiento estaba sometido a una densidad inferior (7 alevinos $56 \mathrm{~L}^{-1}$ ) y por ende los parámetros de calidad del agua fueron mejores al compararlos con el T2 y T3 (Tabla 1). El resultado puede ser atribuido a un incremento de estrés producto del manejo diario en las unidades experimentales, sumado al cambio de conducta en los alevinos, debido a factores de crecimiento, ruido de maquinarias y mayor disposición de espacio para desplazarse, demandando mayor energía para contrarrestar los factores estresantes. Efectos similares en el mismo contexto reportan Ramos et al. (2009); Cifuentes et al. (2012); Sierra (2012).

La producción de biomasa final es variable entre tratamientos $(\mathrm{P}<0.05)$, produciendo hasta $136.9 \mathrm{~kg}$ $\mathrm{m}^{-3}$, resultados muy superiores a los reportados por Del Risco et al., (2008), que obtuvieron una producción de $7.3 \mathrm{~kg} \mathrm{~m}^{-3}$ de biomasa final en alevinos de paiche, criados con densidades de 1 alevino $50 \mathrm{~L}^{-1}$ en condiciones de laboratorio, durante 63 días experimentales, así como a los obtenidos por Tello et al. (2006), con una producción de $26 \mathrm{~kg} \mathrm{~m}^{-3}$ año $0^{-1}$ en juveniles de paiche en jaulas flotantes. Los resultados son incluso superiores a los reportados por Ono \& Kehdi (2013), con $66.36 \mathrm{~kg} \mathrm{~m}^{-3} \mathrm{de}$ biomasa final en paiches criados durante un año, en un sistema de recirculación continua de agua en tanques de PVC. No se registró mortalidad ni canibalismo durante el periodo total de evaluación.

\section{VALORES HEMATOLÓGICOS}

En relación a los valores hematológicos, los resultados se muestran en la Tabla 3, y se presentan bajo el efecto de la densidad, la edad y la interacción entre densidad por la edad. Durante el periodo de evaluación, la densidad no causó variación estadística $(\mathrm{P}>0.05)$. Los valores de hemoglobina registrados en este estudio son levemente inferiores a los reportados por Tavares et al. (2007) y Fonseca et al. (2010) en cultivo semiintensivo, quienes reportan valores de 34 y $33.8 \%$ en juveniles y alevinos de paiche respectivamente. Sin embargo, son ligeramente superiores a los valores de $25.2 \%$ en alevinos de 30 a 180 días de edad obtenidos por Serrano et al. (2013). Asimismo, Ranzani \& Romagoza (2005); Centeno et al. (2007) y Garay \& Paredes (2012), presentan el $28.8 \%$ de hematocrito en Colossoma macropomum, $24.3 \%$ en Pseudoplatystoma fasciatum tomados del ambiente natural y $22.9 \%$ en paco Piaractus brachypomus, todos ellos criados en un sistema semi intensivo.

Los valores promedios de hemoglobina en los tres tratamientos son cercanos a los encontrados por Serrano et al. (2013) en alevinos de paiche, con un valor de $8.67 \mathrm{~g} \mathrm{~d} \mathrm{~L}^{-1} ; 9.1 \mathrm{~g} \mathrm{dL}^{-1}$, registrado por Fonseca et al. (2010) y $10.4 \mathrm{~g} \mathrm{~d} \mathrm{~L}^{-1}$ en juveniles, según Tavares et al. (2007). Sin embargo, al comparar con otras especies amazónicas los resultados difieren, tal es el caso de los alevinos de Pseudoplatystoma fasciatum, con $5.4 \mathrm{~g} \mathrm{~d} \mathrm{~L}$ Ranzani \& Romagoza (2005) y Garay \& Paredes (2011), o los $6.37 \mathrm{~g} \mathrm{~d} \mathrm{~L}^{-1}$ en los alevinos de Piaractus brachypoтus.

En cuanto al factor edad los valores hematológicos mostraron variación $(\mathrm{P}<0.05)$. La interacción entre la densidad por edad no muestra variación estadística $(\mathrm{P}>0.05)$. 
El incremento del hematocrito a los 108 días (33 días experimentales) y su posterior descenso al final de la evaluación, puede estar relacionado a un proceso de adaptación, requiriendo más glóbulos rojos para hacer frente a los factores estresantes, adaptándose luego al nuevo medio, características fisiológicas innatas del Arapaima gigas, que se desarrolló para adaptarse y sobrevivir a los cambios bruscos medioambientales de la Amazonía. Sin embargo, el factor edad también genera variación en los valores hematológicos, Tavares et al. (2007), así como otros factores tales como el requerimiento de oxígeno, concentración de oxígeno y alta difusión de gases en el agua, alimentación, densidad, bacterias y parásitos según Catellanos et al. (2003); Asmine \& Díaz (2004); Centeno et al. (2007); Sanz et al. (2001); Soivio (1982) citado por Barandica \& Tort (2008); Tavares et al. (2009) y Fonseca et al. (2010).

\section{PERFIL BIOQUÍMICO SANGUÍNEO}

La Tabla 4 muestra los resultados del perfil bioquímico sanguíneo de los factores de densidad, edad y la interacción entre la densidad por la edad. En cuanto al factor densidad, la glucosa, triglicéridos y aspartato aminotransferasa no muestran variación $(\mathrm{P}>0.05)$ entre tratamientos. Sin embargo, la proteína, albumina, colesterol y alanina aminotransferasa presentan diferencia significativa $(p<0.05)$ en este mismo factor. Con respecto al factor edad las concentraciones de glucosa, proteína, colesterol, triglicéridos y alanina aminotransferasa presentan diferencias estadísticas significativas $(\mathrm{P}<0.05)$, entretanto, las concentraciones de albúmina y aspartato aminotransferasa no presentan variación estadística $(\mathrm{P}>0.05)$. La interacción entre la densidad por la edad no presentan variación estadística $(\mathrm{P}<0.05)$ en el perfil bioquímico sanguíneo, con excepción de la proteína, que es estadísticamente significativa $(\mathrm{P}<0.05)$ y se muestra en la Tabla 5.

Las concentraciones de glucosa en los tres tratamientos son estadísticamente iguales con respecto a la densidad y cercanas a lo obtenido por Brandão et al. (2004) en alevinos de paiche, con una concentración de $88,9 \mathrm{mg} \mathrm{dL}^{-1}$; Fonseca et al. (2010) $61.9 \mathrm{mg} \mathrm{dL}^{-1}$ en cultivos semi intensivos. El incremento progresivo de la glucosa con respecto a la edad, en especial en el T3, responde al requerimiento de más energía en los alevinos con mayor densidad, para hacer frente a los factores estresantes producto de la densidad y suplir las demandas de energía en el metabolismo, debido a un incremento de almacenamiento de tejido en relación al crecimiento y es registrado en estudios realizados por Tavares et al. (2007) que evaluó juveniles de paiche con pesos promedios de $790 \mathrm{~g}$ y reporta concentraciones de $152.4 \mathrm{mg} \mathrm{dL}^{-1}$ de glucosa.

La densidad y edad causaron variación estadística $(\mathrm{P}<0.05)$ en las concentraciones de proteína, demandando mayor concentración en el T3 con respecto a la densidad y edad, alcanzando una concentración de $3.4 \mathrm{~g} \mathrm{dL}^{-1}$ en la interacción (Tabla 5). Las concentraciones de proteína en los tres tratamientos se acercan a los datos obtenidos en cultivo semi-intensivo por Fonseca et al. (2010), quien indica $1.99 \mathrm{~g} \mathrm{dL}^{-1}$ en alevinos y $3.5 \mathrm{~g} \mathrm{dL}^{-1}$ en juveniles de $2.35 \mathrm{~kg}$ y difieren a los reportados por Tavares et al. (2007) en juveniles de paiche alimentados con $40 \%$ de proteína y peso de $734 \mathrm{~g}$, indica una concentración de $6.5 \mathrm{~g} \mathrm{dL}^{-1}$.

En los resultados obtenidos es evidente el incremento en el requerimiento de proteína para contrarrestar el efecto de la densidad y se registra en el T3 (136.89 $\mathrm{kg} \mathrm{m}^{-3}$ ); asimismo, el incremento de ese nutriente para transformarlo en tejido mediante el metabolismo explica el incremento conforme avanza la edad; también está relacionado con la pérdida de agua a nivel celular por concentración de solutos y a la deficiencia de oxígeno que condiciona la oxidación de la proteína. Un factor muy importante a considerar es la interacción conjunta en los peces de los sistemas nervioso, endocrino e inmune que pudieron activar los mecanismos de defensa ante los efectos de la densidad, el mismo que compromete más concentración de proteína en la sangre, Rubio (2010).

En el caso de la albúmina existe variación estadística $(\mathrm{P}<0.05)$ con respecto a la densidad, entretanto, que la edad no hizo variar las concentraciones. Las bajas concentraciones de albúmina en los alevinos son debidas a una baja presión sanguínea, relacionadas a la baja masa corporal y baja concentración de inmunoglobulinas que poseen, Herrera (2004). Las concentraciones de albúmina en el suero son un buen indicador de la salud hepática. No existen datos referenciales disponibles para este indicador en paiche, sin embargo, los datos registrados en este estudio pueden servir de referencia para trabajos futuros.

En relación al colesterol existe variación $(\mathrm{P}<0.05)$ en relación a la densidad, las concentraciones son inferiores en los tres tratamientos obtenidos por Tavares et al. (2007), quien indica una concentración de $204.1 \mathrm{mg} \mathrm{dL}^{-1}$ en juveniles de paiche en un sistema semi intensivo. Fonseca et al. (2010), registra concentraciones de $280.2 \mathrm{mg} \mathrm{dL}^{-1}$ en alevinos y $255.8 \mathrm{mg} \mathrm{dL}^{-1}$ en juveniles de paiche en el mismo sistema de cultivo. Las concentraciones de triglicéridos en los tres tratamientos no fueron influenciados por la densidad $(\mathrm{P}>0.05)$, son superiores a los reportados por Fonseca et al. (2010) en alevinos de paiche, reportando $73.5 \mathrm{mg} \mathrm{dL}^{-1}$; el 
mismo autor reporta en juveniles una concentración de $397.6 \mathrm{mg} \mathrm{dL}^{-1}$, entretanto, que Tavares et al. (2007) en juveniles reporta concentraciones de $308.1 \mathrm{mg} \mathrm{dL}^{-1}$. La variación en las concentraciones de colesterol y triglicéridos se relacionan con la preferencia que tienen los peces al utilizar los triglicéridos para generar energía, y a la presencia de enzimas comprometidas en el metabolismo de los triglicéridos y cambios fisiológicos propios de la edad según Hepher (1993) y Castellanos et al. (2003). La mayor concentración de triglicéridos comparado a los conseguidos por los autores mencionados se puede atribuir a la calidad del alimento suministrado, frecuencia de alimentación y al sistema de cultivo.

Los resultados obtenidos muestran que no existe diferencia estadística significativa $(\mathrm{P}>0.05)$ entre tratamientos en las concentraciones de aspartato aminotransferasa (AST) con respecto a los factores densidad y edad. Las concentraciones de alanina aminotransferasa $(\mathrm{ALT})$ son variables $(\mathrm{P}<0.05)$. A los 138 días de edad los tres tratamientos muestran un descenso en la concentración de esta enzima. La AST y ALT son los indicadores más eficientes en cuanto a las alteraciones hepáticas. Aun no existe literatura disponible que reporten datos referenciales de estas enzimas en paiche.

Estudios realizados en trucha arco íris Oncorhynchus mykiss, reportan concentraciones de 4,83 $\mathrm{U} \mathrm{l}^{-1}$ de AST según Herrera (2004); mientras que Marcano (2011) encontró 4,83 $\mathrm{U}^{-1}$ de ALT en paco Piaractus brachypomus. Con respeto al descenso de la ALT, Herrera (2004) menciona que esta enzima se incrementa cuando existen daños hepáticos principalmente, pero al mismo tiempo es directamente proporcional al incremento de la AST. Según los resultados las concentraciones de ALT disminuye y no existe incremento considerable en la AST, lo que permite descartar daños hepáticos producto de la densidad y la pésima calidad de agua. Las concentraciones de ALT y AST reportados en otras especies son bajos comparados a los obtenidos en este estudio; sin embargo por los datos obtenidos entre tratamientos y en ambas enzimas, se puede afirmar que estas concentraciones son rangos propios de la especie.

\section{CONCLUSIONES}

La densidad de cultivo no causó efecto sobre los parámetros biométricos de consumo de alimento, ganancia de peso e incremento de talla, con excepción de la conversión alimenticia que fue mejor. El factor de condición fue inferior en el tratamiento con menor densidad, cultivándose hasta $136.9 \mathrm{~kg} \mathrm{~m}-3$ de biomasa final. Los valores hematológicos de hematocrito y hemoglobina, así como las concentraciones de glucosa, triglicéridos y AST no fueron influenciados por la densidad. Las concentraciones de proteína, albúmina y colesterol fueron incrementadas en los alevinos criados con alta densidad. La concentración de ALT fue baja en los alevinos criados con alta densidad. Los parámetros hematológicos y bioquímicos, a excepción de la albúmina y la enzima AST en este estudio, variaron con respecto a la edad. Los resultados obtenidos en cada parámetro biométrico, hematológico y bioquímico, sumado a la baja calidad del agua, ratifican la enorme potencialidad de esta especie para su cultivo intensivo y a gran escala.

Tabla 1. Características de los parámetros fisicoquímicos del agua (mínimos y máximos) en alevinos de paiche sometidos a tres densidades de cultivo, durante 63 días experimentales.

\begin{tabular}{lcccccc}
\hline \multirow{2}{*}{ Parámetros } & \multicolumn{5}{c}{ Densidades (alevinos/56 litros) } \\
\cline { 2 - 7 } & \multicolumn{2}{c}{ T1 (7 alevinos) } & T2 (14 alevinos) & \multicolumn{2}{c}{ T3 (21 alevinos) } \\
\cline { 2 - 7 } & Min & Max & Min & Max & Min & Max \\
\hline Amonio (ppm) & 0.1 & $>8$ & 2.5 & $>8$ & 2.8 & $>8$ \\
\hline Nitrito $(\mathrm{ppm})$ & 0.05 & 0.1 & 0.06 & 0.15 & 0.05 & 0.8 \\
Oxígeno disuelto ${ }^{1}(\mathrm{ppm})$ & 0.8 & 4.1 & 0 & 3.1 & 0 & 2.3 \\
CO $_{2}(\mathrm{ppm})$ & 3 & 34 & 5 & 39 & 14 & 57 \\
Alcalinidad $(\mathrm{ppm})$ & 200 & 330 & 208 & 360 & 215 & 370 \\
\hline Dureza $(\mathrm{ppm})$ & 44 & 68 & 48 & 72 & 55 & 78 \\
pH & 7 & 7 & 7 & 7 & 6 & 7 \\
Temperatura $\left({ }^{\circ} \mathrm{C}\right)$ & 26 & 26 & 26 & 26 & 26 & 26 \\
\hline
\end{tabular}

Leyenda: Mínimo: Min, Máximo: Max. 
Tabla 2. Peso, talla (inicial y final) y parámetros biométricos de crecimiento de alevinos de paiche sometidos a tres densidades de crianza, durante 63 días experimentales.

\begin{tabular}{lccccc}
\hline \multirow{2}{*}{ Parámetros } & \multicolumn{5}{c}{ Densidad (alevinos/56 litros) } \\
\cline { 2 - 6 } & T1 (7 alevinos) & T2 (14 alevinos) & T3 (21 alevinos) & C.V (\%) & P \\
\hline PPI (g) & $69.7 \pm 6.56 \mathrm{a}$ & $83.23 \pm 6.49 \mathrm{a}$ & $71.87 \pm 5.36 \mathrm{a}$ & 16.65 & 0.82 \\
PPF (g) & $363.8 \pm 11.6 \mathrm{a}$ & $402.4 \pm 13.7 \mathrm{a}$ & $361.63 \pm 17.5 \mathrm{a}$ & 8.30 & 0.52 \\
TPI (cm) & $22.1 \pm 0.71 \mathrm{a}$ & $23.53 \pm 0.62 \mathrm{a}$ & $22.43 \pm 0.51 \mathrm{a}$ & 5.38 & 0.75 \\
TPF (cm) & $37.4 \pm 0.28 \mathrm{a}$ & $37.80 \pm 0.44 \mathrm{a}$ & $36.76 \pm 0.46 \mathrm{a}$ & 2.33 & 0.24 \\
BI (kg m-3) & $9.9 \pm 1.24 \mathrm{c}$ & $18.86 \pm 2.28 \mathrm{~b}$ & $27.66 \pm 2.99 \mathrm{a}$ & 6.57 & 0.0001 \\
BF kg m-3 & $47.7 \pm 1.44 \mathrm{c}$ & $97.13 \pm 3.91 \mathrm{~b}$ & $136.89 \pm 6.55 \mathrm{a}$ & 8.38 & 0.0001 \\
CDA (g) & $4.43 \pm 0.24 \mathrm{a}$ & $4.84 \pm 0.24 \mathrm{a}$ & $4.74 \pm 0.25 \mathrm{a}$ & 5.26 & 0.13 \\
GPD (g) & $4.71 \pm 0.14 \mathrm{a}$ & $4.95 \pm 0.14 \mathrm{a}$ & $4.62 \pm 0.24 \mathrm{a}$ & 7.77 & 0.52 \\
IDT (cm) & $14.98 \pm 0.53 \mathrm{a}$ & $14.69 \pm 0.30 \mathrm{a}$ & $14.17 \pm 0.44 \mathrm{a}$ & 4.27 & 0.23 \\
CAA & $0.94 \pm 0.05 \mathrm{a}$ & $0.97 \pm 0.05 \mathrm{a}$ & $1.02 \pm 0.03 \mathrm{~b}$ & 5.26 & 0.04 \\
K & $0.67 \pm 0.01 \mathrm{~b}$ & $0.74 \pm 0.01 \mathrm{a}$ & $0.73 \pm 0.01 \mathrm{a}$ & 2.30 & 0.02 \\
S (\%) & 100 & 100 & 100 & & \\
\hline
\end{tabular}

Letras diferentes dentro de la fila, indica diferencia estadística $(P<0.05)$.

Leyenda: Coeficiente de variación C.V, Peso promedio inicial: PPI, Peso promedio final: PPF, Biomasa inicial: BI; Biomasa final: Bl, Consumo de alimento diario: CAD, Ganancia de peso diario: GPD, Incremento de talla: IDT, Conversión alimenticia aparente: CAA, Factor de condición: K, Sobrevivencia: S.

Tabla 3. Valores de hematocrito y hemoglobina en alevinos de paiche sometidos a tres densidades de crianza durante 63 días experimentales.

\begin{tabular}{llc}
\hline \multirow{2}{*}{ Factores } & \multicolumn{2}{c}{ Parámetros (promedio \pm desviación estándar) } \\
\cline { 2 - 3 } & Hematocrito (\%) & Hemoglobina (g/dL) \\
\hline Densidad & $\mathrm{P}=0.71$ & $\mathrm{P}=0.71$ \\
Edad & $\mathrm{P}=0.0001$ & $\mathrm{P}=0.0008$ \\
Densidad x edad & $\mathrm{P}=0.33$ & $\mathrm{P}=0.89$ \\
\hline C.V (\%) & 6.5 & 7.94 \\
\hline Densidad & & $8.33 \pm 0.19 \mathrm{a}$ \\
\hline T1 (7 alevinos) & $30.58 \pm 1.14 \mathrm{a}$ & $8.43 \pm 0.27 \mathrm{a}$ \\
T2 (14 alevinos) & $29.93 \pm 0.75 \mathrm{a}$ & $8.56 \pm 0.23 \mathrm{a}$ \\
\hline T3 (21 alevinos) & $30.25 \pm 0.80 \mathrm{a}$ & $9.03 \pm 0.12 \mathrm{a}$ \\
\hline Edad (días) & & $7.84 \pm 0.19 \mathrm{~b}$ \\
\hline $75^{1}$ & $29.75 \pm 0.54 \mathrm{~b}$ & $8.45 \pm 0.22 \mathrm{ab}$ \\
\hline $108^{2}$ & $33.33 \pm 0.70 \mathrm{a}$ & 7.93 \\
\hline $138^{3}$ & $27.60 \pm 0.41 \mathrm{c}$ &
\end{tabular}

Letras diferentes dentro de la columna indican diferencia estadística $(\mathrm{P}<0.05)$

Leyenda: Coeficiente de variación: C.V 1, 2, $3=0$, 33, 63 días experimentales respectivamente. 
Tabla 4. Concentraciones promedios del perfil bioquímico sanguíneo en función a la densidad, edad (días experimentales) y la interacción del factor densidad por edad en alevinos de paiche sometidos a tres densidades de crianza durante 63 días experimentales.

\begin{tabular}{|c|c|c|c|c|c|c|c|}
\hline \multirow[b]{2}{*}{ Factores } & \multicolumn{7}{|c|}{ Parámetros } \\
\hline & $\begin{array}{l}\text { Glucosa } \\
\text { mg dL }^{-1}\end{array}$ & $\begin{array}{l}\text { Proteína } \\
\text { total gdL-1 }\end{array}$ & $\begin{array}{l}\text { Albúmina } \\
\mathrm{mg} \mathrm{dL}^{-1}\end{array}$ & $\begin{array}{c}\text { Colesterol } \\
\mathrm{mg} \mathrm{dL}^{-1}\end{array}$ & $\begin{array}{l}\text { Triglicéridos } \\
\mathrm{mg} \mathrm{dL}^{-1}\end{array}$ & $\begin{array}{l}\text { AST } \\
\text { UI L }^{-1}\end{array}$ & $\begin{array}{l}\text { ALT } \\
\text { UI L-1 }\end{array}$ \\
\hline Densidad & $P=0.75$ & $P=0.0182$ & $P=0.0443$ & $P=0.0163$ & $P=0.5363$ & $P=0.7686$ & $P=0.0037$ \\
\hline Edad & $P=0.0084$ & $P=0.0001$ & $P=0.1981$ & $P=0.0117$ & $P=0.0009$ & $P=0.5154$ & $P=0.0010$ \\
\hline D x edad & $P=0.6233$ & $P=0.0155$ & $P=0.3686$ & $P=0.2663$ & $P=0.6733$ & $P=0.5182$ & $P=0.0743$ \\
\hline C.V & 31.2 & 12.65 & 15.16 & 29.11 & 42.18 & 18.34 & 31.13 \\
\hline \multicolumn{8}{|l|}{ Densidades } \\
\hline $\mathrm{T} 1$ & $50.7 \pm 4.82 \mathrm{a}$ & $2.5 \pm 0.1 \mathrm{ab}$ & $0.9 \pm 0.03 \mathrm{ab}$ & $167.6 \pm 16.7 \mathrm{a}$ & $a b 275.5 \pm 43.1 \mathrm{a}$ & $9.9 \pm 0.5 \mathrm{a}$ & $13.3 \pm 1.5 \mathrm{a}$ \\
\hline $\mathrm{T} 2$ & $54.7 \pm 6.11 \mathrm{a}$ & $2.3 \pm 0.1 b$ & $0.8 \pm 0.04 b$ & $125.9 \pm 5.3$ & b $229.4 \pm 35.7$ a & $10.2 \pm 0.5 \mathrm{a}$ & $13.3 \pm 1.3 \mathrm{a}$ \\
\hline T3 & $55.6 \pm 5.16 \mathrm{a}$ & $2.7 \pm 0.2 \mathrm{a}$ & $0.9 \pm 0.04 a$ & $182.2 \pm 19.6$ & a $239.9 \pm 29.1$ a & $9.7 \pm 0.5 a$ & $8.5 \pm 1.2 b$ \\
\hline C.V & $31.19 \mathrm{a}$ & 12.64 & 15.16 & 29.11 & 42.18 & 18.34 & 31.12 \\
\hline \multicolumn{8}{|l|}{ Edad (días) } \\
\hline $75^{1}$ & $40.3 \pm 1.57 b$ & $2.0 \pm 0.1 b$ & $0.91 \pm 0.03 \mathrm{a}$ & $123.3 \pm 13.1 \mathrm{~b}$ & $143.5 \pm 13.2 b$ & $9.5 \pm 0.5$ a & $13.0 \pm 1.2 \mathrm{a}$ \\
\hline $108^{2}$ & $59.9 \pm 6.02 a$ & $2.7 \pm 0.1 \mathrm{a}$ & $0.81 \pm 0.03 a$ & $177.8 \pm 19.9 \mathrm{a}$ & $314.8 \pm 32.5 \mathrm{a}$ & $10.4 \pm 0.5 \mathrm{a}$ & $14.0 \pm 1.5 \mathrm{a}$ \\
\hline $138^{3}$ & $60.7 \pm 5.03 a$ & $2.8 \pm 0.2 \mathrm{a}$ & $0.84 \pm 0.04 a$ & $174.6 \pm 16.5 \mathrm{a}$ & $286.6 \pm 36.3 a$ & $9.9 \pm 0.5 a$ & $8.1 \pm 1.1 b$ \\
\hline
\end{tabular}

Letras diferentes dentro de la columna, indica diferencia estadística $(P<0.05)$;

Leyenda: Coeficiente de variación: C.V, Significancia: P, Densidad: D 1, 2, $3=0$,

33, 43 días experimentales respectivamente, ALT: Alanina aminotransferasa,

AST: Aspartato aminotransferasa.

Tabla 5. Interacción de la densidad por la edad para la proteína, en alevinos de paiche sometidos a tres densidades de crianza, durante 63 días experimentales.

\begin{tabular}{cccc}
\hline \multirow{2}{*}{ Densidad } & \multicolumn{3}{c}{ Edad (días) } \\
\cline { 2 - 4 } & $\mathbf{7 5}$ & $\mathbf{1 0 8}$ & $\mathbf{1 3 8}$ \\
\hline T1 (7 alevinos) & $2.00^{\mathrm{Ab}}$ & $2.00^{\mathrm{Ab}}$ & $2.00^{\mathrm{Ab}}$ \\
T2 (14 alevinos) & $2.65^{\mathrm{Aa}}$ & $2.63^{\mathrm{Aa}}$ & $2.71^{\mathrm{Aa}}$ \\
T3 (21 alevinos) & $2.75^{\mathrm{ABa}}$ & $2.30^{\mathrm{Bab}}$ & $3.39^{\mathrm{Aa}}$ \\
\hline
\end{tabular}

Letras diferentes dentro de la misma columna y/o fila indica diferencia estadística $(\mathrm{P}<0.05)$.

Leyenda: Columnas: $A B$, Filas: ab. 


\section{BIBLIOGRAFÍA CITADA}

Alcántara, F.; Wust, H.; Tello, S.; Rebaza, M. 2006. Paiche el gigante del Amazonas. Instituto de Investigaciones de la Amazonía Peruana. Iquitos, Perú. 16pp.

Aldea, M. 2002. Cultivo de paiches Arapaima gigas (Cuvier, 1982) con dietas artificiales en jaulas flotantes. Tesis de pre-grado. Universidad Nacional de la Amazonía Peruana. Iquitos, Perú. 67 pp. 2

Asmine, B.; Díaz, B. 2004. Parámetros hematológicos de la paragua, Chaetodipterus faber (Broussonet) (Pices: Ephippidae), en condiciones de cultivo. Zootecnia Tropical. Vol. 22:4-10

Barandica, L.; Tort, L. 2008. Neuroendocrinología e inmunología de la respuesta al estrés en peces. Revista Académica Colombiana de Ciencias, Vol. 32: 267-284.

Blanco, A. 2005. Efectos adversos del amoníaco en los acuarios. ). Acceso: 19/11/2013.

Brandão, F.; Gomes, L.; Chagas, E. 2004. Respostas de estresse em pirarucu (Arapaima gigas) durante práticas de rotina em piscicultura. Acta Amazónica. Vol. 36(3).

Castellanos, A.; Bustos, B.; Hernández, A.; Mocha E. 2003. Valoración hematológica y química sanguínea del yamú brycon, en tres etapas de cultivo. Red de Revistas Científicas de América Latina, el Caribe, España y Portugal, 7:34-41.

Centeno, L.; Silva, R.; Barrios, R.; Salazar, R.; Matule, C.; Pérez, J. 2007. Características hematológicas de la cachama Colossoma macropomum en tres etapas de crecimiento cultivadas en el estado Delta Amacuro, Venezuela. Zootecnia Tropical. Vol 25(4).

Cifuentes, R.; González, J.; Montoya, M.; Jara, A.; Ortíz, N. 2012. Relación longitud-peso y factor de condición de los peces nativos del río San Pedro, Chile). Gayana Concepc, Vol. 75(2). 3

Collier, H. 1944. The standardization of blood haemoglobin determinations. Journal List. Can Med Assoc, 50: 550-552.

Crescêncio, R.; Ituassú, D.; Roubach, R.; Pereira F. 2005. Influencia do periodo de alimentação no consumo e ganho de peso do pirarucú. Pesquisa Agropecuaria. Brasileira. Vol.40 (1217-1222).

Del Risco, M.; Velásquez, J.; Sandoval, M.; Padilla, P.; Mori, L.; Chu-Koo, F. 2008. Efecto de tres niveles de proteína dietaria en el crecimiento en el crecimiento de juveniles de paiche, Arapaima gigas. Folia Amazónica. Vol. 17 (1-2).

Fer. D. 2011. Bacterias nitrificantes. (www.Acuariovida.foro.com). Acceso: 12/12/2013.
Fonseca, D.; Caixeiro, A.; Tavares M.; Marcon, J.; Affonso, E. 2010. Características bioquímicas e hematológicas do pirarucú Arapaima gigas Schinz, 1822 (Arapaimidae) de cultivo semiintensivo na Amazônia. Acta Amazónica. Vol. $40(3-8)$.

Franco, H.; Peláez, M. 2007. Contribución al conocimiento de la reproducción del pirarucù Arapaima gigas. Universidad de la Amazonía. Caquetá, Colombia. 50pp.

Garay, M.; Paredes, D. 2011. Caracterización hematológica del paco Piaractus brachypomus (characidae) en tres etapas de crecimiento (alevinos, juveniles y adultos) bajo condiciones de cultivo en el distrito de José Crespo y Castillo. Tesis de pre-grado, Universidad Nacional Agraria de la selva - Tingo María. $65 \mathrm{pp} .4$

Granado, A. 2000. Efecto de la densidad de cultivo sobre el crecimiento del morocoto, Piaractus brachypomus, Cuvier, 1818, (Pisces: Characiformes), confinado en jaulas flotantes. Saber. Vol. 12 (2-6).

Hahn-Von, C.; Grajales, A.; Gutiérrez, A. 2011. Parámetros hematológicos de Tilapia nilótica Oreochromis niloticus (Linnaeus 1757) con peso entre 250 gy $350 \mathrm{~g}$, en el Centro Experimental Piscícola de la CM. Revista vet.zootec. Vol 5(48-50).

Hepher, B. 1993. Nutrición de peces comerciales en estanques. Balderas, México. 81pp.

Herrera, E. 2004. Perfil metabólico de salmón atlántico salmo salar y trucha arcoíris Oncorhynchus mykiss de tres pisciculturas en fase de agua dulce en el sur de Chile. Tesis de pre-grado. Universidad Austral de Chile Facultad de Ciencias Veterinarias. Valdivia, Chile. 19pp.

Marcano, R. 2011. Determinación de parámetros bioquímicos y análisis estructural del riñón cefálico del pez Colossoma macropomum expuesto a cadmio. Tesis de post-grado. Universidad de Oriente Núcleo de Sucre. Cumaná, Venezuela. 45pp.

Ocampo, A.; Ocampo, L. 1999. Diagnóstico del estrés en peces. Red de Revistas Científicas de América Latina, el Caribe, España y Portugal, 30: 337-344.

Ono E.; Kehdi J. 2013. Manual de boas práticas de produção do pirarucu em cativeiro. Serviço Brasileiro de Apoio às Micro e Pequenas Empresas. Brasilia, Brasil. 26pp. 5

Padilla, P., García, A.; Sandoval, M. 2006. Crecimiento compensatorio de alevinos paiche Arapaima gigas, en ambientes controlados. In: 
Renno, J.; Dávila, C; Duponchelle, F.; Nuñez, J. (Eds). Biología de la Poblaciones de peces de la Amazonía y Piscicultura.p.173-177.

Padilla, P.; Isminio, R.; Alcántara, F.; Tello, S. 2003. Producción y manejo de alevinos de paiche en ambientes controlados. In: Alcántara, F. \& Montreuil V. (Eds). Seminario Taller Internacional de Manejo de Paiche o Pirarucu. p. $125-141$.

Padilla, P.; Ismiño, R.; Alcántara, F.; Tello, S. 2006. Efecto de la tasa de alimentación en el crecimiento del paiche Arapaima gigas. In: Alcantara, F., Mntreuil, V.; (Eds). Memoria del seminario Taller Internacional de Manejo de Paiche o pirarucú.p. 125-141.

Ranzani, T.; Romagoza, E. 2005. Hematological parameters of "cachara", (Pseudoplatystoma fasciatum) LINNAEUS, 1766 (Osteichthyes, pimelodidae), Reared In Captivity. Inst. Pesca, São Paulo, 31: 47-53.

Ruales, D.; Arturo, C.; Torres, W. 2009. Transporte iónico en el epitelio branquial de peces de agua dulce. Revista Lasallista de Investigación, 7: 8599.

Rubio, M. 2010. Teleost fish immunology. Revista Mexicana de Ciencias Pecuarias, 1:43-57.

Sagratzki, B.; Pereira-Filho, M.; Roubach, R.; Rabello, D.; Lima A.; Crescêncio, R. 2003. Efeito da densidade de estocagem na homogeneidade do crescimento de juvenis de pirarucú em ambiente confinado. Pesq. agropec. Bras, 38:103-107. 6

Sanz, A.; Alvarez, M.; Hidalgo, M.; Domezain, J.; Morales, A.; García, G. 2001. Constantes eritrocitarios en peces de cultivo intensivo en agua dulce. Universidad de Granada. Granada, España.

Serrano, E.; Leguía, G.; Quispe, M. 2013. Valores hematológicos del paiche Arapaima gigas de la Amazonía peruana. Revista de Investigación Veterinaria del Perú, 24: 248-251.
Sierra, R. 2012. El ruido como fuente de estrés para los peces de cultivo. International Aquafeed, 12:23-34.

Tavares, D.; Frascá, S.; Moraes, F.; Campos, F. 1999. Características hematológicas de teleósteos brasileiros. IV. Parâmetros eritroleucométricos, trombométricos glicemia do matrinxã Brycon cephalus Günther, 1869. Ars Veterinaria, 15:149-153.

Tavares, M.; Barcellos, M.; Marcon, J.; Menezes, G.; Ono, E. 2007. Hematological and biochemical parameters for the pirarucú Arapaima gigas, 1822. Electronic Journal of Ichthyology, 2: 61 - 68.

Tavares, M.; Tenani, R.; Gioli, L.; Faustino, C. 2009. Hematología: ferramenta para o monitoramento do estado de saúde de peixes em cultivo. Revista Brasil. Zoo I. 16 (2): 423 - 431.

Tello, S.; Valdivieso, M.; Rebaza, M.; Rebaza C.; Alcántara, F.; Chu-K, F. 2006. Análisis económico de la crianza de paiche Arapaima gigas en jaulas flotantes a partir de los resultados obtenidos en el lago de Imiría, Ucayali. In: Memoria institucional del IIAP 2006, p: 34.7

Toro, F; Pablo, C; Fernando, G. 2012. Efectos de tres densidades de siembra y disponibilidad de alimento sobre el desarrollo y sobrevivencia de larvas de yaque Leiarius marmoratus. Revista de Sistema de Producción Agroecológica: Vól. 3: (19-24).

Tórrez, W; Hernandez, G; Gutiérrez, M; Yossa, M. 2012. Efecto del nivel de proteína dietaria sobre el crecimiento y parámetros séricos en cachama blanca Piaractus brachypomus. Revista Colombiana de Ciencias Pecuarias. Vol. 25 (450-461).

Wiener Lab. 2008. Método colorimétrico para la determinación de proteínas totales y albúmina en suero. (www.Wiener Laboratorios S.A.I.C. com). Acceso 13/12/2013. 